\title{
Effect of Surfactant HLB Value on Settlement Stability of Magnetorheological Fluid
}

\author{
Yanwen Jiang, Yiping Luo, Hongjuan Ren, Dan Wei \\ College of Automotive, Shanghai University of Engineering Science, Shanghai, China \\ Email: 835401036@qq.com
}

How to cite this paper: Jiang, Y.W., Luo, Y.P., Ren, H.J. and Wei, D. (2018) Effect of Surfactant HLB Value on Settlement Stability of Magnetorheological Fluid. Open Access Library Journal, 5: e5004

https://doi.org/10.4236/oalib.1105004

Received: October 30, 2018

Accepted: November 10, 2018

Published: November 13, 2018

Copyright $\odot 2018$ by authors and Open Access Library Inc.

This work is licensed under the Creative Commons Attribution International License (CC BY 4.0).

http://creativecommons.org/licenses/by/4.0/

\section{cc (i) Open Access}

\begin{abstract}
The hydrophilic-hydrophobic balance (HLB) of surfactants has a great influence on surfactants. Among them, polyethylene glycol has an HLB value of 20 and is highly hydrophilic; oleic acid has an HLB value of 1 , which is very lipophilic; and sodium dodecylbenzenesulfonate has an HLB value of 10.6 between the two. This paper explores the use of these three surfactants with typical HLB values to improve the performance of magnetorheological fluids, mainly to study its effect on the stability of magnetorheological fluids. Tests have shown that the use of lipophilic surfactants in oil-based magnetorheological fluids has better sedimentation stability than the use of hydrophilic surfactants.
\end{abstract}

\section{Subject Areas \\ Composite Material}

\section{Keywords}

Magnetorheological Fluid, Surfactant, Sedimentation Stability, HLB Value

\section{Introduction}

The magnetorheological fluid is a stable suspension that can be controlled by a magnetic field by mixing magnetic particles having magnetization properties in a base liquid. It has a unique magnetic field controllable property, which is a Newtonian fluid state without an external magnetic field; in the state of an external magnetic field, the magnetorheological fluid immediately transforms into a solid-like state, and its conversion degree is within a range of variation and magnetic field strength [1]. It is positively correlated, and once the applied magnetic field is removed, the magnetorheological fluid immediately becomes a Newtonian fluid with a change time of milliseconds [2] [3]. At present, various 
types of magnetorheological fluid devices have been used in shock absorbers, brakes, vibration absorption devices for bridges and buildings, surface polishing devices for optical components, and medical devices. As a new type of intelligent material, magnetorheological fluid has broad engineering application prospects and can play its important value in many fields such as vehicle engineering, mechanical engineering, environmental protection, medical, aerospace engineering, military and so on [4] [5].

\section{Settlement Stability of Magnetorheological Fluid}

At present, there are many problems in the study of magnetorheological fluids. Among them, the problem to be solved is the settlement and agglomeration of solid magnetic particles of magnetorheological fluid. Since the magnetorheological fluid is composed of a base liquid, dispersed particles, and various types of additives. Under normal circumstances, in the case where magnetic particles are used as the dispersed particles, the density of the magnetic particles, such as the density of the most common carbonyl iron powder, is about $7.8 \mathrm{~g} / \mathrm{cm}^{3}$, and the density of the base liquid, such as the more common types of silicone oil. And mineral oil, synthetic oil, the density is basically floating above $1 \mathrm{~g} / \mathrm{cm}^{3}$, so the density of magnetic particles is much larger than the density of the base liquid, so the overall stability of the magnetorheological fluid is low, and the magnetorheological fluid is inevitable over time. Settling and agglomeration will occur [6]. We can calculate the sedimentation velocity of magnetic particles in magnetorheological fluid according to the Stokes formula:

$$
V=\frac{2 d^{2}\left(\rho-\rho_{0}\right) g}{9 \eta}
$$

In the above formula, $d$ is the particle size of the magnetic particles; $\rho$ is the density of magnetic particles; $\rho_{0}$ is the density of the carrier liquid; $g$ is the acceleration of gravity; $\eta$ is the viscosity of the carrier liquid.

\section{Effect of Surfactants on the Settlement Stability of Magnetorheological Fluid}

Surfactants are currently commonly used to improve the stability of magnetorheological fluids [7]. Since the surfactant is amphiphilic, and the most commonly used dispersed particles in the magnetorheological fluid are carbonyl iron powder, the carbonyl iron powder is a hydrophilic substance, so when the carbonyl iron powder is dispersed into the lipophilic base liquid (such as silicone oil) as well as various types of mineral oils and synthetic oils, serious separation occurs, and even obvious condensation and agglomeration occur. When the surfactant is sufficiently contacted with the carbonyl iron powder, the hydrophilic group at one end of the surfactant molecule is adsorbed on the surface of the carbonyl iron powder, and the lipophilic group at the other end is diffused into the base liquid. The lipophilic group can diffuse into the carrier liquid like a whip. The Brownian motion of the particles in the carrier liquid also causes 
these whip tips to entangle each other. These intertwined whip tips can form a spatial three-dimensional framework structure in the base fluid, thereby reducing particle settling due to the difference in density between the particles and the base fluid. At the same time, the surfactant is coated on the surface of the carbonyl iron powder, which increases the specific surface area of the carbonyl iron powder, and increases the repulsive force between the particles, thereby reducing the agglomeration between the particles [8] [9].

HLB (Hydrophile-Lipophile Balance Number) value: Surfactant is a molecule composed of hydrophilic and lipophilic groups. It has both hydrophilic and lipophilic properties. The size and balance of hydrophilic and lipophilic groups of surfactants the degree is the hydrophilic-lipophilic balance (HLB value) of the surfactant. The HLB value is called the hydrophilic hydrophobic equilibrium value, also called water oiliness. It was first proposed by W.C. Griffin in 1949, indicating the equilibrium relationship between the hydrophilic group and the lipophilic group in the surfactant molecule [10].

The hydrophilic and hydrophobic equilibrium value of the surfactant has a great influence on the surfactant. The hydrophilicity or lipophilicity of the surfactant can be judged by the value of the HLB value. The larger the HLB value, the more hydrophilic, the smaller the HLB value is. Oils, in general, surfactants have HLB values between 1 and 40. The HLB value is not only related to the hydrophilic and lipophilic properties of the surfactant, but also affects the surface properties, adsorption, emulsifying properties and emulsion stability, dispersibility, solubility, and detergency of the surfactant. At the same time, the application properties of the surfactant are determined. Therefore, it has important reference value in practical applications. In general, the transition point of the hydrophilic-lipophilic property of the surfactant has an HLB value of 10, and when the HLB value is less than 10, the surfactant exhibits lipophilic properties, and when the HLB value is greater than 10 , the surfactant exhibits hydrophilic character.

When Griffin proposed the concept of HLB value in 1949, the HLB value of the nonionic surfactant was set to 0 - 20. Among them, since the paraffin oil consists entirely of saturated alkane groups, it is completely oleophilic, and the HLB value is determined. It is 0 ; while polyoxyethylene is composed of a hydrophilic oxyethylene group, which is completely hydrophilic, and has an HLB value of 20; other surfactants have an HLB value of between 0 and 20. The smaller the HLB value, the stronger the lipophilicity; the larger the HLB value, the stronger the hydrophilicity. With the advent of new surfactants, more hydrophilic varieties have been used, such as the HLB value of $40 \%$ sodium lauryl sulfate.

The surfactant applied to the magnetorheological fluid has a hydrophilic group adsorbed on the surface of the magnetic particle, and the lipophilic group is easily diffused in the carrier liquid, so that the magnetic particle can be better dispersed in the carrier liquid. The hydrophilic-hydrophobic balance (HLB) of surfactants has a great influence on surface activity. 


\section{Experiment}

\subsection{Experimental Materials}

The magnetic particles are micron-sized carbonyl iron powder, the carrier liquid is dimethyl silicone oil, the thixotropic agent is nano-silica, and the lubricant is graphite. The purpose of this experiment was to investigate the effect of different HLB values on the magneto-rheological fluid settling, so three surfactants with large differences in HLB values were selected. Polyethylene glycol: its HLB value is 20; sodium dodecylbenzenesulfonate has an HLB value of 10.6; oleic acid: its HLB value is 1 .

Experimental instruments: KQM-Z/B model planetary ball mill; DZF-6020 model vacuum drying oven; JJ-1 precision booster electric mixer.

\subsection{Production of Magnetorheological Fluid}

There are many ways to prepare magnetorheological fluids [11]. This paper mainly uses secondary ball milling method based on base fluid replacement. A certain amount of absolute ethanol was mixed with the surfactant, stirred uniformly, and the weighed carbonyl iron powder was poured into the mixed solution, stirred well, and then ball milled in a ball mill for 12 hours. The ball-milled suspension was dried in a vacuum oven at a vacuum of $-1 \mathrm{mPa}$ and a temperature of $60^{\circ} \mathrm{C}$. Weigh a certain amount of nano-silica, graphite and dimethyl silicone oil, mix and stir well, mix with the dried carbonyl iron powder, stir well, place the suspension in a ball mill, and grind for 8 hours to obtain magnetic Rheology fluid (Table 1).

\subsection{Anti-Settling Performance Test}

The magnetic properties of the magnetic particles were measured by a vibrating sample magnetometer; the sedimentation stability was obtained by visual observation of the stratification of the magnetorheological fluid base liquid and the turbid liquid, and the height percentage of the clear liquid layer was measured over time at intervals changes. Settlement stability is evaluated by sedimentation rate:

$$
V=a /(a+b) \times 100 \%
$$

In the above formula: $a$-the height of the supernatant. $b$-the height of the lower turbid liquid.

Table 1. Composition and content of magnetorheological fluid additives (\%).

\begin{tabular}{cccc}
\hline Sample & Polyethylene glycol & SDBS & Oleic acid \\
\hline MRF-0 & 0 & 0 & 0 \\
MRF-1 & 1 & 0 & 0 \\
MRF-2 & 0 & 1 & 1 \\
MRF-3 & 0 & 0 & 1 \\
\hline
\end{tabular}




\subsection{Experimental Results and Analysis}

When the surfactant was not used, the sedimentation of the magnetorheological fluid was the most serious, and the surfactant-free MRF-0 had a sedimentation amount of $14.8 \%$ in two weeks, which was significantly inferior to the magnetorheological fluid to which the surfactant was added. This is because the surfactant is adsorbed on the surface of the carbonyl iron powder particles, which can be regarded as the volume of the iron powder becomes larger, thereby reducing the density of the iron powder, reducing the density difference between the iron particles and the carrier liquid, and finally making the surface active. The addition of the agent enhances the sedimentation stability of the magnetorheological fluid.

Comparing the sedimentation relationship of different surfactant magnetorheological fluids, the sedimentation stability of the magnetorheological fluid with oleic acid added is optimal, and the sedimentation amount after two weeks is $11.2 \%$. The magnetorheological fluid added with sodium dodecylbenzenesulfonate settled to about $12.15 \%$ after two weeks, and $14.1 \%$ when added with polyethylene glycol. This can be attributed to the fact that polyethylene glycol is completely hydrophilic. It adsorbs well on the surface of carbonyl iron powder, but its dispersibility in silicone oil is weaker than sodium dodecylbenzenesulfonate and oleic acid. Oleic acid can form a good coating even though the hydrophilic group is less, and the carbonyl iron powder is well melted in the silicone oil. Therefore, the sedimentation property of the magnetorheological fluid to which oleic acid is added is superior to the magnetorheological fluid to which polyethylene glycol is added. In summary, the use of surfactants can improve the stability of magnetorheological fluids, but for different carrier liquids, it is important to choose a surfactant based on the HLB value of the surfactant, which is better. Improve the sedimentation stability of the magnetorheological fluid.

\section{Conclusions}

1) The use of surfactant can significantly improve the sedimentation stability of magnetorheological fluid;

2) The use of a small HLB value in the oil-based carrier liquid, that is, the lipophilic surfactant is more effective for improving the stability of the magnetorheological fluid.

\section{Conflicts of Interest}

The authors declare no conflicts of interest regarding the publication of this paper.

\section{References}

[1] Rabinow, J. (1948) The Magnetic Fluid Clutch. Electrical Engineering, 67, 1308-1315.

[2] Barnes, H.A., Hutton, J.R. and Walters, K. (1998) An Introduction to Rheology. El- 
sevier, Amsterdam.

[3] Phulé, P.P. and Ginder, J.M. (1998) The Materials Science of Field-Responsive Fluids. MRS Bulletin, 23, 19-21. https://doi.org/10.1557/S0883769400030761

[4] Dyke, S.J., Spencer, B.F. and Sain, M.K. (1998) An Experimental Study of MR Dampers for Seismic Protection. Smart Materials and Structures, 7, 693-703. https://doi.org/10.1088/0964-1726/7/5/012

[5] Jolly, M.R., Bender, J.W. and Carlson, J.D. (1998) Properties and Applications of Commercial Magnetorheological Fluids. SPIE, 3327, 262-275.

[6] Phulé, P.P., Mihalcin, M.P. and Genc, S. (1999) The Role of the Dispersedphase Remnant Magnetization on the Redispersibility of Magnetorheological Fluids. Journal of Materials Research, 14, 3037-3041. https://doi.org/10.1557/JMR.1999.0407

[7] Kin, M.S., Cho, M.S. and Choi, H.J. (2007) PMMA Coated Carbonyl Iron Microbeads and Their Magneticcharacteristics. Physica Status Solidi (a), 204, 4198-4201. https://doi.org/10.1002/pssa.200777351

[8] Batchelor, G.K. (1977) Effect of Brownian-Motion on Bulk Stress in a Suspension of Spherical-Particles. Journal of Fluid Mechanics, 83, 97-117. https://doi.org/10.1017/S0022112077001062

[9] Bossis, G., Volkova, O., Lacis, S. and Meunier, A. (2002) Magnetorheology: Fluids, Structures and Rheology. In: Odenbach, S., Ed., Ferrofluids, Springer, Berlin, 202-230. https://doi.org/10.1007/3-540-45646-5_11

[10] Shinoda, K. and Saito, H. (1969) The Stability of O/W Type Emulsions as Functions of Temperature and the HLB of Emulsifiers: The Emulsification by PIT-Method. Journal of Colloid \& Interface Science, 30, 258-263. https://doi.org/10.1016/S0021-9797(69)80012-3

[11] Charles, S.W. (2002) The Preparation of Magnetic Fluids. In: Odenbach, S., Ed., Ferrofluids, Springer, Berlin, 3-19. https://doi.org/10.1007/3-540-45646-5_1 